\title{
Deep Vein Thrombosis in Lower Extremity of a Female Collegiate Volleyball Athlete: A Case Study
}

Lucas D. Dargo, DAT, LAT, ATC*; Kendra Borton, LAT, ATC; Rachel Roths, LAT, ATC; and Zachary Dougal, DAT, LAT, ATC $\dagger$

*Manchester University, North Manchester, IN †Ball State University, Muncie, IN

\section{ABSTRACT}

Deep vein thrombosis (DVT) occurs when a blood clot forms in deep veins in the body, usually in the lower extremity. DVT is commonly seen in older or hospitalized patients. This case is unique because the patient is a 20 -year-old female division III collegiate volleyball athlete, which is not the typical age or population affected by DVT or clotting disorders. The patient presented with swelling, pain, and decreased knee flexion (3/5) and plantarflexion (4/5) strength in her left lower extremity. These impairments reduced her ability to walk or run. The patient's athletic trainer (AT) initially diagnosed her with a left gastrocnemius muscle strain, resulting in contraindicated treatments. The AT referred the patient to the team physician, who referred her to the Emergency Department (ED) where she received a real-time grayscale and Doppler ultrasound of the left lower extremity deep venous structures, leading to the DVT diagnosis. The patient received a dose of Lovenox, an intravenous anticoagulant, by the ED physician. The following day, she began $15 \mathrm{mg}$ of Xarelto, twice per day for 21 days, to dissolve the blood clots. She could not participate in volleyball for approximately two weeks, which reduced her ability to socialize with teammates and coaches. The Wells' scoring system is a clinical prediction tool to identify patients with low risk of being diagnosed with DVT. The AT had a low suspicion of DVT and therefore did not use the Wells' scoring system, likely leading to a delay in the diagnosis and treatment. Although the patient reported that she had a positive outcome, ATs should be familiar with the Wells Clinical Prediction Rule. Blood clots in veins can dislodge and travel through the bloodstream. The loose clot can block blood flow in the lungs, forming a pulmonary embolism. Early diagnosis can prevent this life-threatening condition.

Key Phrases

Diagnostic testing, lower extremity, general medical conditions

\section{Correspondence}

Dr. Lucas Dargo, Manchester University, 604 E. College Avenue North Manchester, IN 46962.

E-mail: Iddargo@manchester.edu

Twitter: @lucasdargo

\section{Full Citation}

Dargo L, Borton K, Roths R, Dougal Z. Deep vein thrombosis in lower extremity of a female collegiate volleyball athlete: A case study. Clin Pract Athl Train. 2020;3(2):55-62. https://doi.org/10.31622/2020/0002.8.
Submitted: April 7, 2019 Accepted: March 15, 2020

\section{INTRODUCTION}

$\mathrm{V}$ enous thrombosis is a condition that includes deep vein thrombosis (DVT) and pulmonary embolism (PE). An annual incidence rate of about 1 per 1000 adults, ${ }^{1}$ which rapidly increases after the age of 45.2 Incidence rates are slightly higher in men than women, and approximately two-thirds of episodes manifest as DVT, while one-third as a PE with or without DVT. ${ }^{3}$ DVT occurs when a blood clot forms in one or more of the deep veins in the body, usually forming in the lower extremity. However, it can also occur in the upper extremities. ${ }^{4}$ This disease typically occurs after surgical procedures and trauma in the presence of malignancy or inherited coagulation disorders, but can present without any apparent etiologic event. ${ }^{5}$ Approximately half of all DVT cases occur in hospitalized patients or nursing home residents. ${ }^{6}$ Symptoms of DVT include tachycardia, pyrexia, leg pain and tenderness, swelling, and dilation of the superficial veins.

The Well's Clinical Prediction Rule, commonly known as the Well's scoring system, is a valid, nine-item, clinical prediction rule for DVT. This clinical prediction rule previously demonstrated excellent interobserver reliability (Kappa = $0.85),{ }^{8}$ developed as a safe and feasible pretest with probability for DVT. 9 It is critical for ATs to be familiar with these 9 questions so they may consider using this tool if a patient presents with signs and symptoms consistent with DVT, particularly when the patient presents with three or more signs and symptoms. The number and location of the clots, patient's age, and absence of trauma created a unique case of DVT. In this 
case study, the patient first received a misdiagnosis due to the lack of suspicion.

\section{Patient Information}

This case follows a 20-year-old female Division III collegiate volleyball player. A timeline of this case is provided (Figure 1). On August 14, 2017, the patient reported to the athletic training facility (ATF) with a swollen lower left leg and discomfort with walking. The team's AT performed the initial evaluation. During this evaluation, the patient reported to the athletic trainer (AT) that she had no previous history of DVT. The patient revealed to the clinician that she took Propafenone-Ethinyl Estradiol (YAZ) 3-0.02 mg, a daily oral contraceptive medication. The patient reported that she had previously diagnosed with pes planus a few months earlier in the summer preceding camp, and received a walking boot for two weeks. Furthermore, she stated that a week before reporting to the ATF, her uninvolved leg had swelled, but reduced within a couple of days.

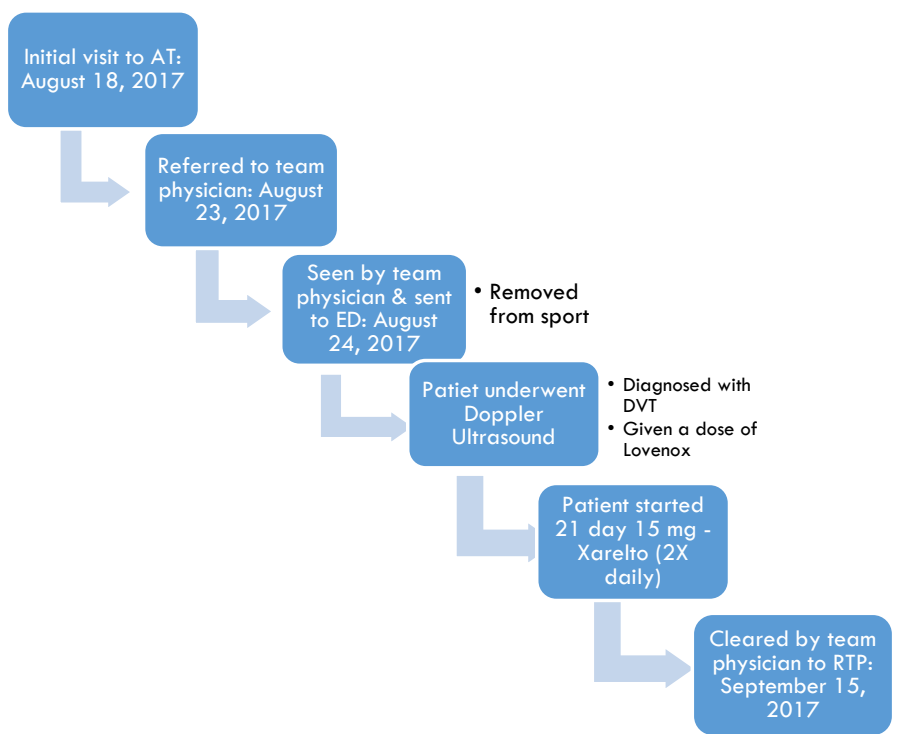

Figure 1. Timeline of Care; $A T$, athletic trainer; ED, emergency department; RTP, return-to-play

Copyright $($ ) by Indiana State University All rights reserved. ISSN Online 2577-8188

\section{Differential Diagnosis and Evaluation}

After four days of treatment, the patient did not progress as the AT had expected for a gastrocnemius muscle strain. During this time, the patient returned-to-play without restrictions. The team physician later referred her for additional diagnostic testing due to her calf swelling and difficulty with walking. During his evaluation, the team physician referred her to the hospital for a Doppler ultrasound to rule out DVT. The patient drove to the ED via her mother's personal vehicle. After being admitted to the ED, the patient's mother reported to the ED physician that the patient had a factor $V$ Leiden deficiency, an inherited blood-clotting disorder due to a mutation of the blood's factor $V$ protein. Furthermore, the patient received a Doppler ultrasound, positive for DVT.

The patient had three blood clots, one in each of her left femoral vein, popliteal vein, and the profundal vein. The Wells' scoring system (Table 1) would have revealed a score of 3 at the initial evaluation; one point given due to her recent immobilization of the walking boot, one point given for her entire left leg swelling, and localized tenderness along the distribution of the deep venous system of her left lower extremity. Due to the low suspicion for DVT, there were no measurements of calf girth or the use of the Homan's sign to rule in or rule out DVT. Due to the location of pain, obvious swelling, use of contraceptive, suspicion for DVT should have been raised and the use of the Wells' score would have suggested a high probability for a DVT diagnosis. 
Body Structure and Function

Manual muscle testing determined the severity within the left lower extremity: $3 / 5$ knee flexion, $5 / 5$ knee extension, $5 / 5$ dorsiflexion, and $4 / 5$ plantarflexion. The patient reported with knee flexion and plantarflexion limitations. Once the patient received the correct diagnosis of DVT, the focus of her treatment changed from the musculoskeletal system to the circulatory system. The ED physician referred the patient to a hematologist for additional care. The patient reported that she still had pain throughout the range of motion of the left knee.

\section{Activity and Participation}

During the initial evaluation, the patient could not walk without pain and had an observable limp. Functional tests were determined to be unnecessary to perform during this time. The AT witheld the patient from practice following the initial evaluation and established the goal to help control the patient's pain and swelling. Two days later, the patient returned to the AT without the need for crutches. Although the patient's calf still had some swelling, she returned to full practice participation for two days before she had to discontinue activity due to pain. During this time, the patient received treatment: 20-minutes of compression cryotherapy, seven minutes of a milk massage, instrument-assisted soft tissue mobilization, bike or treadmill warm up, and abdominal core workouts.

Following the DVT diagnosis, the patient received a single dose of Lovenox to dissolve her blood clots. The ED physician discharged her the same day as arrival. She returned to her dorm room, where she elevated the affected area above the level of her heart when sitting. She wore a compression stocking and applied a warm compress or heating pad to the affected area as directed by the ED physician. The physician encouraged the patient to avoid prolonged standing or bed rest, avoid smoking, to discontinue taking Meloxicam, and her oral contraception.

Copyright (C) by Indiana State University All rights reserved. ISSN Online 2577-8188
Table 1. Wells criteria for the prediction of deep vein thrombosis $(D V T)^{10,11}$

\section{Clinical Characteristic}

Score

Active cancer (patient either receiving 1 treatment for cancer within the previous 6 months or currently receiving palliative treatment)

Paralysis, paresis, or recent cast immobilization of the lower extremities

Recently bedridden for $\geq 3$ days, or major surgery within the previous 12 weeks requiring general or regional anesthesia

Localized tenderness along the distribution of the deep venous system

Entire leg swelling

Calf swelling at least $3 \mathrm{~cm}$ larger than that on the asymptomatic side (measured 10 $\mathrm{cm}$ below tibial tuberosity)

Pitting edema confined to the symptomatic leg

Collateral superficial veins (non-varicose) $\quad 1$

$\begin{array}{ll}\text { Previously documented DVT } & 1\end{array}$

Alternative diagnosis at least as likely as $\quad-2$ DVT

Wells scoring system for DVT: -2 to 0 : low probability, 1 to 2 points: Moderate probability, 3 to 8 points: High probability

After this time, the ED physician allowed her to return to activities of daily (ADL). The physician did not provide a specific progression for ADLs. Once the patient discontinued the $15 \mathrm{mg}$ of Xarelto, taken twice a day for 21 days the physician determined the patient no longer had a risk for developing a PE. After that time, the patient returned to volleyball without restriction. 
Currently, there is not an established return-toplay protocol for individuals recovering from a venous thromboembolism. However, it is critical that providers develop a structured program of gradually increased activity as tolerated by the patient. ${ }^{12}$ Roberts and Christie report a case of a female triathlete with acute lower extremity DVT that suggested a structured, gradual return-totraining protocol (Table 2). ${ }^{13}$ In the first three weeks, the gradual introduction of $A D L s$ is suggested, while patients complete their anticoagulation medication. ${ }^{13}$ Once endothelialization and adhesion are achieved, the potential for clot migration and embolism is reduced. ${ }^{12}$ Between the fourth to sixth weeks of this protocol, Roberts and Christie suggest a gradual return-to-training regimen that starts with non-weight-bearing exercises, next cycling, and finally, running. ${ }^{13}$ If the patient reports any previous signs or symptoms returning, the protocol should be discontinued. Moreover, it is important the AT and patient note any bruising. ${ }^{12}$ In this case, the patient completed the first three weeks of this protocol prior to fully return to play (RTP). Additional sport-specific activities should have been gradually integrated prior to full RTP.

Table 2. Post-anticoagulation return-totraining recommendations.

\begin{tabular}{ll} 
Weeks 1-3 & $\begin{array}{l}\text { Gradual return to ADLs } \\
\text { Begin non-weight-bearing } \\
\text { exercises (e.g., swimming) }\end{array}$ \\
Week 5 & $\begin{array}{l}\text { Begin nonimpact-loading } \\
\text { exercises (e.g., cycling) }\end{array}$ \\
Weeks 6+ & $\begin{array}{l}\text { Begin impact-loading } \\
\text { exercises (e.g., begin running } \\
\text { progression) }\end{array}$ \\
\hline
\end{tabular}

\section{Environmental and Personal Factors}

The patient hesitated to schedule the initial evaluation because she worried about losing her spot on the team. Also, she reported that the right calf presented similarly, earlier in the summer but resolved within a couple of days. After several days of pain and inflammation in her left calf causing difficulties with walking, she decided to

Copyright (C) by Indiana State University All rights reserved. ISSN Online 2577-8188 seek help. The patient's pain affected her ability to perform ADLs such as walking, running, and going upstairs. The patient discontinued her oral contraception due to the increased increase risk of DVT.

\section{INTERVENTION}

The patient's AT initially provided treatment for a soft tissue injury, a left gastrocnemius muscle strain. The primary goals of the interventions were to reduce the patient's pain and swelling. Treatments included 20-minutes of compression cryotherapy, seven minutes of a milk massage, instrument-assisted soft tissue mobilization, bike or treadmill warm up, and abdominal core workouts. The AT performed these treatments for three consecutive days. Once the patient received the DVT diagnosis, this course of treatment was discontinued. The massage and soft tissue treatments are contraindicated for a DVT diagnosis because of the potential risk factors for developing a PE by dislodging a blood clot. ${ }^{11}$ After the ED physician diagnosed the patient with DVT they did not suspect an active PE. The ED physician discharged the patient the same day as arrival and instructed her to begin ambulation within 24 hours, elevating her left calf above her heart when seated. The day following discharge, the patient began a 21-day series of twice-daily $15 \mathrm{mg}$ of Xarelto. After the 21 -day series, the patient could perform all ADLs. The team physician cleared the patient for full RTP. To reduce the potential of bleeding, the hematologist ordered the patient to reduce her dosage of Xarelto to one daily dose of $20 \mathrm{mg}$.

The patient was instructed by the team physician to discontinue taking Propafenone-Ethinyl Estradiol (YAZ) 3-0.02 mg because of its known side-effect of increasing the risk for clot formation. ${ }^{14}$ Additionally, the team physician told her to discontinue taking Meloxicam, an acne medication, due to its possible interactions with the Xarelto. The ED physician prescribed the patient $15 \mathrm{mg}$ of Xarelto, administered orally twice daily, 
to treat the pre-existing blood clots and prevent new clots from forming. Following her visit to the hematologist, further testing confirmed that the patient was positive for factor $V$ Leiden mutation. This is an inherited blood-clotting disorder due to a mutation of the blood's factor $V$ protein. ${ }^{15}$ As of January 2019, the patient continued to see both her hematologist and primary care physician several times throughout the year for a physical exam and a comprehensive metabolic panel to monitor the status of the blood clots.

\section{OUTCOMES}

\section{Body Structure and Function}

The Xarelto is expected to continue to shrink the blood clots. This allows the body to naturally dissolve them. The left leg continues to remain swollen. The hematologist deemed this as normal; this condition is referred to as post-thrombotic syndrome. ${ }^{15}$ After 22 months following her diagnosis, the patient is now able to complete full range of motion without pain. Muscle strength returned to pre-injury performance. Strength was accessed by the patient's ability to perform both $A D L s$ and sport participation without any restrictions.

\section{Activity and Participation}

The team's AT monitored the patient for signs and symptoms as she RTP. The team physician followed Roberts and Christie's suggested RTP protocol for the first three weeks, ${ }^{13}$ keeping the patient out of sport participation. Neither the team physician nor the AT initiated a gradual RTP progression after this time. The patient successfully competed in the rest of the volleyball season without her symptoms returning or sustaining any additional injuries.

\section{Environmental and Personal Factors}

The patient reported that she was pleased with how she performed following her RTP. Since her left leg was more swollen than the right, people often asked her if it was safe for her to RTP. This

Copyright (C) by Indiana State University All rights reserved. ISSN Online 2577-8188 caused her to be irritated. The oral contraception medication the patient was taking may have predisposed her the blood clots.

\section{DISCUSSION}

Deep vein thrombosis is a rare and emergent medical condition, especially in young active individuals. ${ }^{14}$ It is critical that ATs are educated properly on the appropriate recognition and management for DVT. The Wells' scoring system is a helpful 9-item clinical diagnostic prediction tool for DVT; ${ }^{6}$ based on yes or no questions. Each question is given a numeric value (Table 1). A score of zero or lower suggests DVT is unlikely (5\%), 1-2 moderate risk (17\%), 3 and above DVT is likely (17-53\%).9,10 An AT should consider using the Wells' scoring criteria if they suspect a patient presents with any combination of signs or symptoms of DVT, such as sudden shortness of breath, chest pain or discomfort that worsens when they take a deep breath or cough, feeling lightheaded or dizzy, have a pulse $>100$ beats per minute, coughing up blood, or having posterior leg pain.

It is critical to ask patients about their current and past use of oral contraceptive medications because some brands and dosages can cause patients to be prone to blood clotting. Moreover, it is critical to determine if patients have a known medical history that includes genetic disorders. Vandenbroucke et al. found that there is an increased risk of DVT in oral contraceptive users who are carriers of factor $V$ Leiden mutation. ${ }^{16}$ Additionally, the AT in this case likely overlooked DVT as a differential diagnosis, due to lack of suspicion because of the patient's young age and active status.

The use of a patient-rated outcome measure, such as the SF-36, should be used throughout a patient's treatment to measure their healthrelated quality of life. The SF-36 would have complemented the use the Wells' scoring system, as it can be used to track the overall quality of life of patients with DVT. ${ }^{17}$ The SF-36 can be used 
to determine when a patient is ready to progress to the next step in the return-to-play DVT progression. ${ }^{13}$ Additionally, patients who develop post-thrombotic syndrome PTS after DVT report poorer health related quality of life using both generic and disease-specific questionnaires. ${ }^{18}$ For example, if the SF-36 was used in this case by the AT, they may have identified that the patient was having difficulty with ADLs as well as their emotional health. Although the patient's care was delayed, this patient was able to return to full participation and ALDs. Although the patient was able to fully return-to-play, as of 22 months post diagnosis the patient continued her treatment of $20 \mathrm{mg}$ of Xarelto, taken once daily. We recommend that ATs consider implementing the Wells' scoring system when patient's present with signs and symptoms consistent with DVT. Although evidence supports anticoagulation and early mobilization, guidelines for return-to-play require additional research. ${ }^{12} \mathrm{We}$ propose ATs use Roberts and Christie's suggested return-to-play protocol (Table 2) and patient-rated outcome measures to determine when DVT patients' activity levels should progress.

\section{CLINICAL BOTTOM LINE}

When evaluating a patient who presents with calf pain, ATs should determine if other signs, symptoms, and risk factors associated with venous thrombosis are present (Figure 2). If warranted, ATs should consider implementing the Wells' scoring criteria. This will aid the clinician in determining if the patient is a low, moderate, or high probability of DVT. If further consultation is needed, ATs should consult with their supervising physician or activate their emergency action plan. Early diagnosis can help prevent the progression of a DVT to a PE. Compression ultrasonography is considered the gold standard to diagnose DVT. ${ }^{19}$ Additional research is needed to determine specific clinical practice guidelines. If the patient is diagnosed with DVT, patient-rated outcome measures should be used in combination with clinician-rated outcome measures to confirm the

Copyright (C) by Indiana State University All rights reserved. ISSN Online 2577-8188 patient's progress. Patients should be instructed to discontinue sport participation if signs or symptoms return. Particular attention should be given to bruising or loss of blood.

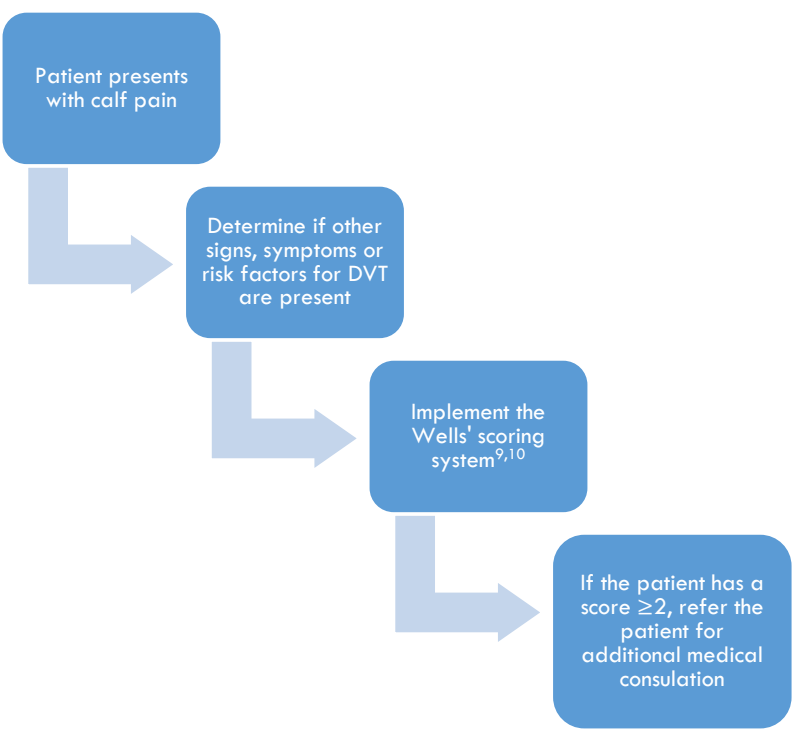

Figure 2. Evaluation process for patient with potential DVT

\section{PATIENT PERSPECTIVE}

The patient agreed to do an interview about her experience. The interview was conducted approximately a year after she returned-to-play. The patient thought the care she received was adequate. She reported that the athletic training staff provided care to the best of their abilities and used the knowledge that was best at the time. The patient was asked what the athletic training staff could do differently about her treatment. She answered, "No milk massage or scraping because it made my leg hurt more." When asked how she felt about her return-to-play, the patient answered, "I wasn't worried. They warned me that my leg would remain swollen. I was more worried about getting other injuries." When asked how the injury affected this year's season, the patient replied, "It didn't really affect this year. My leg still swells. I still have the blood clots, but they are shrinking. I am still on the medication. It'll take time for the clots to go away. The only thing that is different is that I take the medication at night now." 


\section{REFERENCES}

1. White RH. The epidemiology of venous thromboembolism. Circulation. 2003;107(23 Suppl 1):14-8. https://doi.org/10.1161/01.cir.000007846 8.11849 .66 .

2. Tsai AW, Cushman M, Rosamond WD, Heckbert SR, Polak JF, Folsom AR. Cardiovascular risk factors and venous thromboembolism incidence: The longitudinal investigation of thromboembolism etiology. Arch Intern Med. 2002;162(10):1 182-9. https://doi.org/10.1001/archinte.162.10.1 182.

3. Cushman M, Tsai AW, White RH, Heckbert $S R$, Rosamond WD, Enright $P$, et al. Deep vein thrombosis and pulmonary embolism in two cohorts: The longitudinal investigation of thromboembolism etiology. Am J Med. 2004; 1 17(1):19-25. https://doi.org/10.1016/i.amimed.2004.01 .018.

4. Heil J, Miesbach W, Vogl T, Bechstein WO, Reinisch A. Deep vein thrombosis of the upper extremity: A systematic review. Dtsch Arztebl Int. 2017;1 14: 244-9.

https://dx.doi.org/10.3238\%2Farztebl.201 7.0244.

5. Prandoni $P$, Villalta $S$, Bagtella $P$, Rossi L, Marchiori A, Piccioli A, et al. The clinical course of deep vein thrombosis. Prospective long-term follow-up of 528 symptomatic patients. Haematologica. 1997;82:423-428.

6. Silverstein MD, Heit JA, Mohr DN, Petterson TM, O'Fallon WM, Melton LJ, III. Trends in the incidence of deep vein thrombosis and pulmonary embolism: A 25-year populationbased study. Arch Intern Med. 1998; 158(6):585-93. https://doi.org/10.1001/archinte.158.6.58 $\underline{5}$.

7. Yang S-D, Liu H, Sun Y-P, Yang D-L, Shen $Y$, Feng $S-Q$, et al. Prevalence and risk factors of deep vein thrombosis in patients after spine surgery: a retrospective case-cohort study. Scientific Reports. 2015;5(1):1 1834. https://dx.doi.org/10.1038\%2Fsrep1 1834.

8. Wells PS, Hirish J, Anderson DR, Lensing AW, Foster $G$, Kearon $C$, et al. Accuracy of clinical assessment of deep-vein thrombosis. Lancet. 1995:345(8961): 1326-30.

https://doi.org/10.1016/s01406736(95)92535-x.

9. Wells PS, Anderson DR, Bormanis J, Guy F, Mitchell M, Gary L, et al. Value of assessment of pretest probability of deepvein thrombosis in clinical management.

Lancet. 1997;350(9094):1795-8.

https://doi.org/10.1016/s01406736(97)08140-3.

10. Wells PS, Anderson DR, Rodger $M$, Forgie $M$, Kearon C, Dreyer J, et al. Evaluation of Ddimer and the diagnosis of suspected deep vein thrombosis. N Engl J Med. 2003:1 227 1235. https://doi.org/10.1056/NEJMoa023153.

11. Lim DC, Jayanthi HK, Money-Kyrle A, Ramrakha P. Massaging the outcome: an unusual presentation of pulmonary embolism. BMJ Case Rep. 2009; 1505.

https://doi.org/10.1136/bcr.01.2009.1505

12. Depenbrock PJ. Thromboembolic disorders: guidance for return-to-play. Curr Sports Med Rep. $2011 ; 10(2): 78-83$. https://doi.org/10.1249/JSR.0b013e3182 $14 \mathrm{~d} 828$.

13. Roberts WO, Christie DM. Return to training and competition after deep venous calf thrombosis. Med Sci Sports Exerc. 1992;24(1 1:2-5).

14. Atmaca H, Köprülü D, Kiriș T, Zeren G, Șahin I. Simultaneous total occlusion of two coronary arteries associated with use of drospirenone-ethinyl estradiol (oral contraceptive). Turk Kardiyol Dern Ars. 2018;46(1): 69-72. https://doi.org/10.5543/tkda.2017.57606.

15. Schleimer K, Barbati ME, Gombert A, Wienert, V, Grommes J, Jalaie H. The treatment of post-thrombotic syndrome. Dtsch Arztebl Int. 2016;1 13(50):863-870. https://doi.org/10.3238arztebl.2016.0863.

16. Vandenbroucke JP, Koster T, Rosendaal FR, Briet E, Reitsma PH, Bertina RM. Increased risk of venous thrombosis in oralcontraceptive users who are carriers of favor $\checkmark$ leiden mutation. The Lancet. 1994;344(8935):1 1453-1457. 
https://doi.org/10.1016/s01406736(94)90286-0.

17. Kahn SR, Hirsch A, Shrier I. Effect of postthrombotic syndrome on health-related quality of life after deep venous thrombosis. Arch Intern Med. 2002;162(10):1144-8. https://doi.org/10.1001/archinte.162.10.1 144.

18. Ghanima W, Wik HS, Tavoly M, Enden T, Jelsness-Jørgensen LP. Late consequences of venous thromboembolism: measuring quality of life after deep vein thrombosis and pulmonary embolism. Thromb Res.

2018; 164:170-176.

https://doi.org/10.1016/i.thromres.2017.0 7.025.

19. Scarvelis D, Wells PS. Diagnosis and treatment of deep-vein thrombosis. CMAJ. 2006; 175(9): 1087-1092.

https://doi.org/10.1503/cmaj.060366. 